\title{
Evolución de un Sistema de Gestión de Seguridad y Salud en el Trabajo e Impacto en la Accidentalidad Laboral: Estudio de Caso en Empresas del Sector Petroquímico en Colombia
}

\author{
PROGRESS OF AN OCCUPATIONAL HEALTH AND SAFETY MANAGEMENT SYSTEM THAT IMPACTS WORK- \\ PLACE ACCIDENTS: CASE STUDY OF PETROCHEMICAL COMPANIES IN COLOMBIA
}

Martha Isabel Riaño-Casallas', Eduardo Hoyos Navarrete², Ivonne Valero Pacheco ${ }^{3}$

1. Profesora Asociada. Especialización en Gestión de la Seguridad y Salud en el Trabajo, Universidad Jorge Tadeo Lozano, Bogotá, Colombia.

2. Especialista en Salud Ocupacional. Docente Catedrático Especialización en Gestión de la Seguridad y Salud en el Trabajo, Universidad Jorge Tadeo Lozano, Bogotá, Colombia.

3. Especialista en Administración en Salud Ocupacional. Profesora Asociada. Especialización en Gestión de la Seguridad y Salud en el Trabajo, Universidad Jorge Tadeo Lozano, Bogotá, Colombia.

\section{RESUMEN}

Introducción: La adopción de sistemas para la gestión de la salud y seguridad en el trabajo trata de responder a las demandas y presiones de los entes regulatorios, empleadores y trabajadores para garantizar un ambiente de trabajo seguro previniendo los accidentes y reduciendo el número de lesionados. Objetivo: analizar el impacto en la accidentalidad laboral que tiene la implementación de un sistema de gestión de seguridad y salud en el trabajo bajo el estándar OHSAS 18001. Método: se recopilaron los datos de la accidentalidad de cuatro empresas del sector petroquímico, tres años antes y después de la certificación en la norma OHSAS, y se realizó una revisión documental y una entrevista al responsable de seguridad y salud en el trabajo para observar el grado de evolución del sistema de gestión a partir de seis elementos: política, identificación de peligros, objetivos y programas, control operacional, medición del desempeño e investigación de accidentes. Resultados: en relación con los índices de frecuencia, severidad y lesiones incapacitantes de los accidentes, de las cuatro empresas analizadas, no presentan una tendencia clara de disminución en los tres años posteriores a la certificación. Sin embargo, el reporte de observaciones si aumentó en tres de las empresas estudiadas. Conclusión: En general, se evidenció que la evolución del sistema de gestión se ha dado como resultado de los cambios en la normatividad legal y no hay una tendencia clara frente a la disminución de la severidad y frecuencias de los accidentes.

(Riaño-Casallas M, Hoyos E, Valero I, 2016. Evolución de un Sistema de Gestión de Seguridad y Salud en el Trabajo e Impacto en la Accidentalidad Laboral: Estudio de Caso en Empresas del Sector Petroquímico en Colombia. Cienc Trab. Ene-Abr; 18 [55]: 68-72).

Palabras clave: OHSAS 18000, PREVENCIÓN DE ACCIDENTES, SEGURIDAD INDUSTRIAL, INDUSTRIA PETROQUÍMICA (DECS).

\section{ABSTRACT}

Introduction: The adoption of occupational health and safety management systems is a response to the demands and pressures of regulatory agencies, employers and workers to guarantee a safe work environment free that prevents accidents and reduces the number of people injured. Given Colombian companies' tendency to seek certification in those systems, particularly under the OHSAS 18001:2007 standard. Objective: Goal was defined of analyzing the impact on workplace accidents of implementing an occupational health and safety management system under the OHSAS 18001 standard. Method: all the data on workplace accidents was compiled, with ratios of severity, frequency and disabling injury of petrochemical companies three years before and after their OHSAS standard certification. Overall, we found that the progress of the management system has come about as the result of changes in the country's standards, and not so much as a product of continuous improvement processes according to the system's performance results. Results: In relation to accidents rates of frequency, severity and disability injuries, four companies analyzed do not show a clear downward trend in the three years following certification. However, if the report of observations increased in three of the companies studied. Conclusion: we did not find a clear trend toward a decrease in the severity nor the frequency of accidents. However, there has been improvement in terms of observations of unsafe conditions.

Key words: OHSAS 18001, OCCUPATIONAL HEALTH AND SAFETY, MANAGEMENT SYSTEMS, WORKPLACE ACCIDENT, INDUSTRIAL SAFETY, PETROCHEMICAL INDUSTRY.
Correspondencia / Correspondence:

Martha Isabel Riaño-Casallas.

Carrera 4 \# 22 - 61, Bogotá, Colombia.

Tel.: 5712427030 ext. 3680 - 3689.

e-mail: marthai.rianoc@utadeo.edu.co

Recibido: 14 de Noviembre de 2016 / Aceptado: 13 de Marzo de 2016

\section{INTRODUCCIÓN}

La Organización Internacional del Trabajo (OIT) y la Organización Mundial de la Salud (OMS) reiteradamente han hecho llamados a los gobiernos para que establezcan politicas públicas en seguridad y salud laboral que incentiven a los empresarios a invertir en prevención de los accidentes y enfermedades relacionadas con el trabajo, ya que el costo económico y social de esta problemática es muy alto. Esto porque la accidentalidad laboral tiene conse- 
cuencias sobre la productividad y competitividad de las empresas y sobre la sociedad en su conjunto.

En este sentido, como estrategia para la prevención de los riesgos laborales surgen con relativa fuerza sistemas estandarizados de gestión, tales como la norma OHSAS 18001, enfocados particularmente en la gestión de la seguridad y salud en el trabajo. De esta forma, son muchas las empresas que en el mundo han adoptado e implementado estos sistemas con el propósito de mejorar continuamente en el campo de la salud en el trabajo, puesto que la implementación de estos sistemas trata de responder a las demandas y presiones de los entes regulatorios, empleadores y trabajadores para garantizar un ambiente de trabajo seguro previniendo los accidentes y reduciendo el número de lesionados. ${ }^{1}$

En Colombia -por las exigencias normativas, de calidad en los procesos, requerimientos de compañías multinacionales o para la exportación de productos a mercados extranjeros-, la necesidad de contar con un sistema certificado ha aumentado en los últimos años. En el pasado, en el país la gestión de los riesgos laborales implicaba sólo la necesidad de cumplir con la reglamentación en términos de tener un programa de salud ocupacional; sin embargo, a partir del año 2012, se les exige a las empresas implementar un sistemas de gestión con el objetivo de anticipar, reconocer, evaluar y controlar los riesgos que puedan afectar la seguridad y salud en el trabajo. ${ }^{2}$

La Agencia Europea para la Seguridad y Salud en el Trabajo ${ }^{3}$ establece que los sistemas de gestión de seguridad y salud en el trabajo son componentes esenciales de cualquier estrategia destinada a potenciar ambientes de trabajo seguro y saludable. Para la OIT, estos sistemas de gestión tienen por objeto proporcionar un método para evaluar y controlar los riesgos en el trabajo mejorando los resultados en la prevención de accidentes y enfermedades laborales. ${ }^{4}$ De estos sistemas, la norma OHSAS 18001 es el único sistema certificable, razón por la cual es el que tiene más reconocimiento y aceptación en el mundo. ${ }^{5}$

En general, los Sistemas de Gestión de Seguridad y Salud en el Trabajo (SGSST) son una herramienta para el desarrollo de actividades preventivas en la organización, brindando medios para la gestión de la seguridad y la salud de una forma organizada y estructurada (6). Al aplicar dichos sistemas de gestión, la organización puede obtener como resultado una reducción de la accidentalidad, además de un aumento en la productividad, lo cual impacta directamente en los resultados económicos y financieros de la empresa. ${ }^{6}$

El sistema de gestión de seguridad y salud en el trabajo, bajo la norma OHSAS 18001, indica unos requisitos mínimos para la gestión de los riesgos laborales con el fin de mejorar su desempeño en este campo. ${ }^{7}$ Se puede indicar que el sistema de gestión en salud y seguridad, OHSAS 18001, constituye un mecanismo para sistematizar y organizar el trabajo en materia de riesgos, posibilita la conexión entre las distintas áreas de la organización y proporciona técnicas y metodologías para una apropiada utilización de los recursos disponibles, permitiendo el desarrollo de las actividades de la empresa a través del establecimiento de políticas y objetivos. ${ }^{8}$

La norma OHSAS 18001 es un estándar certificable que le permite a la organización garantizarle a sus partes interesadas y terceros que tiene una adecuada gestión de salud y seguridad para control los riesgos, permitiendo fortalecer la relación con los accionistas, acreedores, clientes, sindicatos, aseguradores y el gobierno e incrementar su poder de negociación. ${ }^{9}$
La finalidad de cualquier sistema de gestión es el mejoramiento continuo; por tanto, cuando una empresa inicia un proceso de implementación, mantenimiento y certificación de su sistema de gestión, se espera que en el largo plazo este presente mejores resultados, que para el caso de la seguridad y salud en el trabajo es la reducción de la accidentalidad laboral. De hecho, el estándar OHSAS 18001 busca que las organizaciones certificadas tengan un mejor desempeño de seguridad y menos accidentalidad. ${ }^{10,11}$ Según el objeto y campo de aplicación de la NTC OHSAS 18001:2007, se define que esta es aplicable a cualquier organización que desee: establecer un sistema de gestión en seguridad y salud en el trabajo con el fin de eliminar o minimizar los riesgos para el personal y otras partes interesadas que pueden estar expuestas a peligros de seguridad y salud asociados con sus actividades; e implementar, mantener y mejorar continuamente un sistema de gestión en esta materia. ${ }^{7}$ Este estándar busca soportar y promover las buenas prácticas en salud laboral y seguridad industrial con un proceso de gestión estructurado y sistemático ${ }^{12}$, lo cual implica que cambie y se ajuste a las dinámicas de la organización.

Evaluar el desempeño de la organización frente a su gestión de seguridad y salud en el trabajo puede hacerse con los indicadores tradicionales de accidentalidad ${ }^{13}$, como son frecuencia y severidad de las lesiones ocupacionales; sin embargo, también es necesario ver cómo evoluciona dicho sistema, frente al resultado de tales indicadores.

Es precisamente ese proceso de cambio y evolución del sistema de gestión uno de los principales puntos a evaluar en la gestión de seguridad y salud en el trabajo, ya que los procesos de trabajo, así como los peligros y riesgos a los que están expuestos los trabajadores no son estáticos. La implementación del SGSST en las organizaciones debe responder no solo a unas directrices o líneas guías para la minimización de los riesgos, sino a una herramienta proactiva para el mejoramiento continuo de la gestión de seguridad y salud en el trabajo en las compañías. ${ }^{14}$

De acuerdo con lo expuesto hasta el momento, el Sistema de Gestión de Seguridad y Salud en el Trabajo tiene como fin el control de los riesgos a la salud de las personas, lo que motiva a las empresas a su implementación. Teniendo en cuenta este propósito y ante la falta de conocimiento frente al comportamiento de la accidentalidad de las empresas una vez adoptado dicho sistema, el objetivo de la investigación fue analizar el impacto en la accidentalidad laboral que tiene la implementación de un sistema de gestión de seguridad y salud en el trabajo bajo la norma OHSAS 18001, aplicado en empresas colombianas de diferentes sectores económicos.

El presente artículo está organizado de la siguiente forma. En la segunda parte se describe la metodología utilizada, en la tercera sección se presentan los resultados empíricos y, por último, está la discusión e implicaciones para futuras líneas de investigación.

\section{MÉTODO}

El tipo de investigación fue descriptiva con enfoque cuantitativo y cualitativo. Se realizó el análisis con cuatro casos de empresas del sector petroquímico certificadas en la norma OHSAS 18001. Para la recolección de los datos cuantitativos se tomó información de la accidentalidad laboral correspondiente a tres años antes de la certificación y tres años después, según lo propuesto en el estudio de Hamidi y colaboradores ${ }^{15}$, con el fin de establecer un parámetro 
comparativo de los datos definidos para esta investigación, los cuales fueron el índice de severidad (IS), el índice de frecuencia (IF), el índice de lesión incapacitante (ILI) y el número de reportes de actos y condiciones inseguras (observaciones).

Para la investigación cualitativa se tuvo en cuenta el grado de evolución del sistema de gestión, aspecto que fue evaluado en seis elementos representativos de la norma OHSAS 18001:2007: política de seguridad y salud en el trabajo (SST), identificación de peligros, valoración de riesgos y determinación de controles, objetivos y programas, control operacional-contratistas, investigación de accidentes y medición del desempeño.

Las fuentes de información provienen de tres orígenes: primero, de los registros de accidentalidad laboral de la empresa; segundo, de los documentos del sistema de gestión; y, tercero, de una entrevista semi-estructurada realizada al responsable de seguridad y salud en el trabajo de la empresa. Para la recolección de la información se diseñaron tres instrumentos elaborados por el grupo de investigación, los cuales fueron valorados por expertos en gestión de la seguridad y salud en el trabajo. El primer instrumento fue un "formato de captura de información básica"; se recopiló la información general de la empresa y se consignaron los datos de IF, IS, ILI y observaciones durante tres años antes y después de la certificación. El segundo fue una entrevista semiestructurada, y, el tercero, una lista de chequeo para la revisión documental con el fin de verificar la información suministrada por parte del responsable de seguridad y salud en el trabajo.

El análisis de la información cuantitativa se hizo mediante estadísticas descriptivas de los indices de accidentalidad y para la información cualitativa se realizó una codificación a través del software ATLAS. TI V 6.2.

Durante el desarrollo de la investigación se vincularon ocho empresas; sin embargo, en el proceso de recolección de la información solo cuatro cumplieron con la totalidad de los requisitos solicitados, esto era disponer de estadísticas de accidentalidad tres años antes y tres después de la certificación. En este punto se evidenció que antes de la implementación del sistema de gestión cuatro empresas contaban parcialmente con los reportes de accidentalidad laboral, aun cuando por norma legal todas las empresas del país deben llevar estos registros.

\section{RESULTADOS}

\section{a. Casos estudiados}

En cuanto a la caracterización de las empresas que finalmente se incluyeron en el estudio, las cuatro empresas pertenecen al sector petroquímico y son compañías privadas. En la Tabla 1 se observan cuantos empleados directos y contratistas tiene cada una, así como certificaciones adicionales a OHSAS 18001 con las que cuentan las empresas objeto de estudio. También se indagó acerca de cuál fue
Figura 1.

Índice de Frecuencia (I.F.). - Empresa 1 Empresa 2 Empresa 3 - Empresa 4

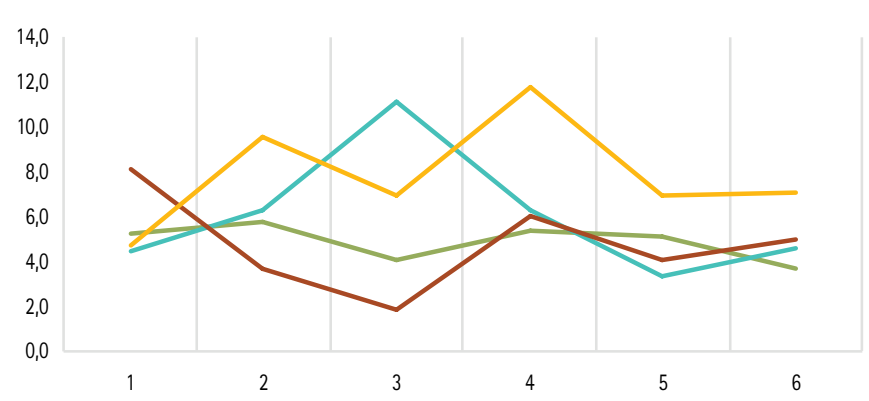

Figura 2.

Índice de Severidad (I.S.).

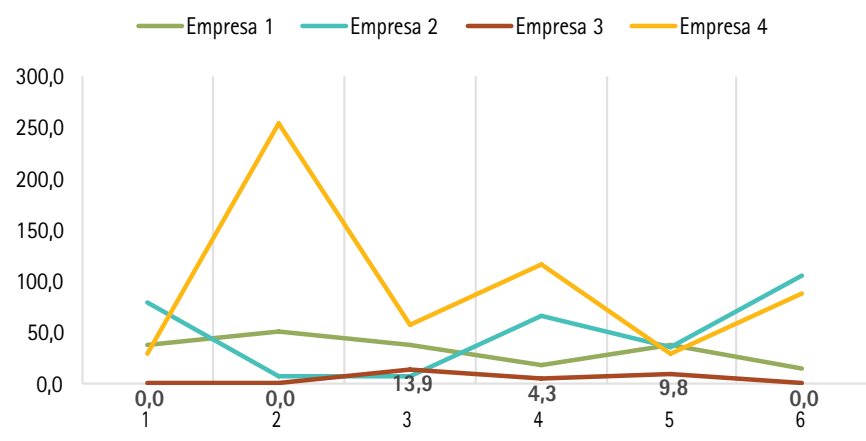

la motivación para certificarse en la norma OHSAS, clasificándolas en interna, cuando hacen referencia a requisitos del cliente, lineamiento de casa matriz, directriz de la gerencia; o externas, si fue por exigencia del sector en el que operan.

\section{b. indicadores de accidentalidad}

A continuación se presentan los hallazgos en cuanto a los indicadores de accidentalidad laboral. En la Figura 1, se detalla el IF, en el cual se observa que este indicador presenta un comportamiento irregular, sin marcar una tendencia desde la obtención de la certificación del SGSST. Las empresas 1 y 3 traían un comportamiento descendente y en el primer año después de certificarse generaron un incremento en el valor del indicador, con un ligero descenso en el año 5. En el caso de las empresas 2 y 4 presentaron incremento en el año de la certificación y el siguiente, respectivamente, denotando un descenso significativo en el siguiente año, el cual se mantiene en los siguientes.

En la Figura 2, se encuentra el IS, el cual también presenta un comportamiento irregular. La empresa 4 es la que muestra un comportamiento más acentuado en los valles y las crestas de los 6 años, pero estos se suavizan a partir de la certificación (año 3). La empresa 2, por el contrario, muestra un incremento a partir de la

Tabla 1.

Caracterización de las empresas incluidas en el estudio.

\begin{tabular}{|c|c|c|c|c|c|c|c|c|c|}
\hline Empresas & Empleados directos & $\%$ & Contratistas & $\%$ & Total de trabajadores & ISO 9001 & ISO 14001 & RUC ${ }^{\circledast}$ & $\begin{array}{l}\text { MOTIVACIÓN PARA } \\
\text { LA CERTIFICACIÓN }\end{array}$ \\
\hline Empresa 2 & 804 & $84.28 \%$ & 150 & $15.72 \%$ & 954 & SI & SI & SI & Externa \\
\hline Empresa 4 & 209 & $89.70 \%$ & 24 & $10.30 \%$ & 233 & SI & SI & NO & Externa \\
\hline
\end{tabular}

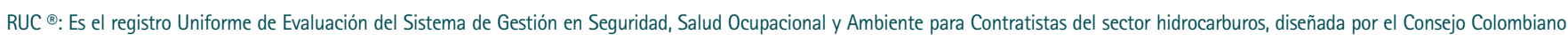
de Seguridad 
Figura 3.

Índice de Lesión Incapacitante (ILI).

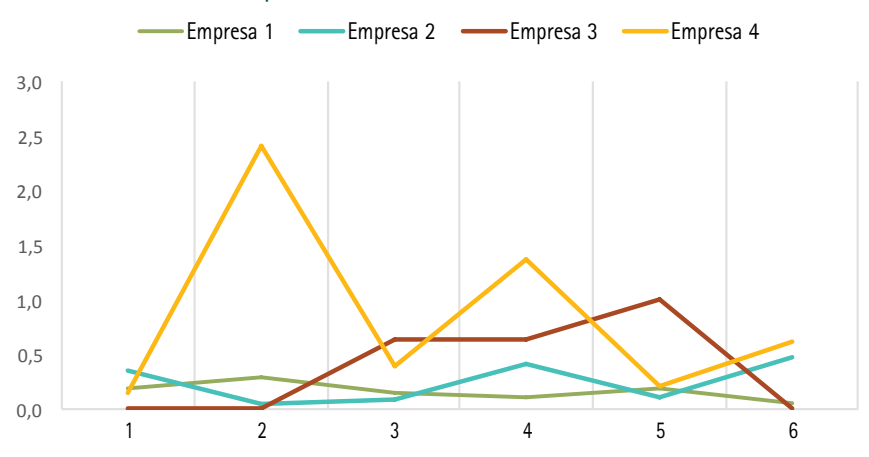

Figura 4.

Reporte de Observaciones.

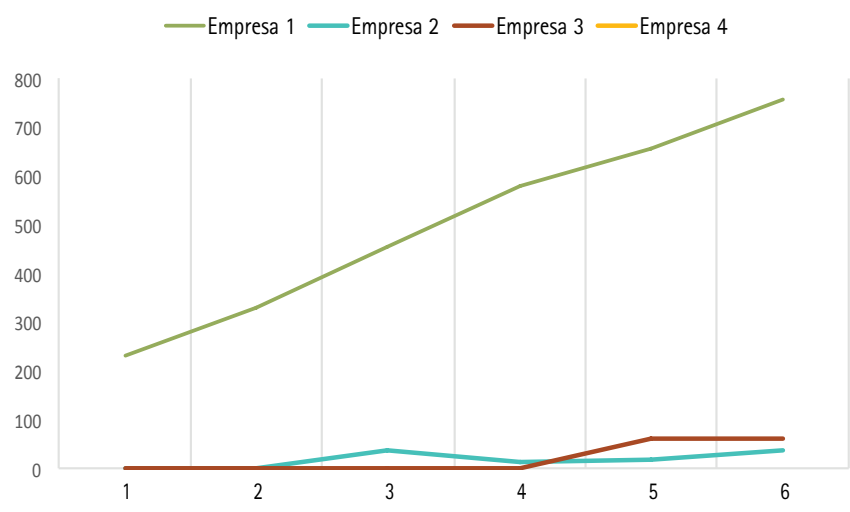

certificación y, en el caso de las empresas 1 y 3, los indicadores son más o menos constantes, con una ligera inclinación al descenso.

El ILI (Figura 3), por ser la combinación de los dos indicadores anteriores, muestra que en la empresa 4 hay un comportamiento similar a su severidad; la 3 presenta un incremento a partir del tercer año, el cual desciende en el sexto. Las empresas 1 y 2 , una secuencia de sube y baje con pendientes muy pequeñas.

Finalmente, en el número de observaciones (Figura 4), la empresa 4 presenta un crecimiento sostenido, el cual puede deberse a la sensibilización de los trabajadores, lo cual aumenta la conciencia y la cultura del reporte. Para el caso de las empresas 2 y 3 se mantiene en el tiempo con un pequeño incremento en los años 5 y 6.

\section{c. Evolución del sistema}

La evolución del sistema de gestión de seguridad y salud en el trabajo, en los casos analizados: el primer elemento abordado fue la política, la cual brinda las orientaciones generales del sistema de gestión y manifiesta el compromiso de la alta dirección con la prevención de los riesgos laborales. De acuerdo con lo referido por los responsables del sistema de gestión, para los cuatro casos de estudio, esta se ha mantenido durante los tres años de la certificación, aunque se han realizado modificaciones derivadas de cambios legales, ajustes en la terminología o en la redacción y suscripción de nuevos compromisos contractuales con clientes, es decir, que su evolución está asociada con ajustes menores.

Para el caso del numeral de identificación de peligros, valoración de riesgos y determinación de controles, se encontró que este proceso ha cambiado una vez se implementan controles operacionales, así como con los resultados de la investigación de acci- dentes, las inspecciones planeadas, los análisis de trabajo seguros, los lineamientos corporativos o el proceso de trabajo. De lo anterior se confirma que este requisito evoluciona a medida que va madurando el sistema.

En relación con los objetivos y programas, las empresas, durante los tres años de la certificación, han incluido y desarrollado nuevos objetivos y programas, teniendo como criterio el grado de desarrollo de los mismos, los resultados de metas e indicadores del sistema, y cambios en la legislación.

El control operacional se revisó considerando únicamente los controles implementados con contratistas, puesto que era una forma de medir el alcance global del sistema de gestión, al incluir las partes interesadas. Además porque, en Colombia, el sector petroquímico está ampliamente tercerizado. $\mathrm{Al}$ respecto se encontró que las modificaciones al SGSST para seguimiento e incorporación de contratistas y terceros fueron las siguientes: evaluación global de contratistas frente a la legislación, establecimiento de requerimientos mínimos para contratistas, inclusión de pautas de contratación con manuales de interventoría y supervisión, inspecciones preoperativas, auditorías de seguimiento, evaluaciones de desempeño, exigencias de los elementos de protección personal, programas de salud ocupacional y pagos de seguridad social.

La investigación de accidentes únicamente se ha modificado frente al cumplimiento de la ley, la inclusión de registros y formatos, y, en un caso, a requisitos contractuales que exigieron el cambio en la metodología de la investigación del incidente.

Finalmente, en cuanto a medición y seguimiento del desempeño del sistema de gestión, los cambios se generan a través de las revisiones gerenciales anuales, donde, producto de estas, las organizaciones incluyeron indicadores de medición de impacto y realizaron ajustes a las metas propuestas. Lo anterior demuestra que en la medida que se va desarrollando el sistema se busca medir su efectividad, más allá del cumplimiento de actividades.

\section{DISCUSIÓN}

Frente al impacto del sistema de gestión en la accidentalidad laboral, en términos de su severidad y frecuencia, para las empresas objeto de estudio no se encontró una clara tendencia a su disminución, como sí lo reportan en los estudios de Vinodkumar y Bhasi ${ }^{10}$, Omran y colaboradores ${ }^{11}$ y Chanh y Liang. ${ }^{13}$ Esto puede explicarse, en parte, porque el periodo de tiempo fue muy corto o porque al evaluar la evolución del sistema se encontró que sus cambios están sujetos a modificaciones normativas y no a mejora continua del mismo sistema.

Por otra parte, aunque se pregunta si tenían personal contratista, al revisar las estadísticas de accidentalidad laboral, estas solo estaban disponibles para el personal contratado directamente con la compañía; no obstante, en el objeto de la norma OHSAS 18001 se dice expresamente que deben involucrarse a todas las partes interesadas, es decir, incluyendo a contratistas. ${ }^{7}$ Además, solo una de las cuatro empresas tiene información relacionada con la accidentalidad de sus contratistas en los proyectos de la compañia objeto del análisis, lo que dificulta evaluar el verdadero impacto de la accidentalidad en las empresas, ya que un alto porcentaje de trabajadores en Colombia actualmente se vincula laboralmente a través de la tercerización.

En cuanto a los reportes y observaciones, estos son necesarios como estrategia de prevención pues ayudan a medir el desempeño 
en seguridad antes de que ocurran los accidentes. ${ }^{10}$ Lo cual sí se observó en la compañías estudiadas, pues los indicadores de reporte de observaciones aumentaron cada año.

En relación con la evolución de los elementos sistema de gestión de seguridad y salud en el trabajo, se encontró que los principales cambios ocurren por las modificaciones en las normas legales, esto particularmente para la política y la investigación de accidentes. Los elementos con menos cambios son los objetivos y programas, y el control operacional, lo cual llama la atención ya que son estos los que permiten en mayor medida el control de la accidentalidad laboral. Y las principales modificaciones se dan en la identificación de peligros y medición y seguimiento al desempeño, puesto que en las empresas estudiadas se incorporaron nuevos indicadores, especialmente de impacto. Sin embargo, no se puede apreciar una evolución clara de los elementos de la norma OHSAS 18001, teniendo en cuenta que no se dispone de suficiente información previa a la implementación del sistema en la organización.

Una de las limitaciones del estudio es que algunas empresas en Colombia, aun cuando por normatividad legal debe llevar el registro de los accidentes laborales, al revisar esta información se evidenció que no se registran o no se mantienen los reportes de estos eventos. No obstante, cuando la empresa decide certificarse mejoran sus sistemas de información y la cultura del reporte.
Por último, es necesario continuar con estudios longitudinales, incluyendo más de tres años en el análisis posterior a la certificación, esto porque entre más tiempo se puede ver mejor la evolución e impacto de la accidentalidad del sistema, lo cual puede enriquecer la investigación, así como incluir otros contextos geográficos y firmas que operen en varias industrias.

\section{Agradecimientos}

Los autores agradecen a la Universidad Jorge Tadeo Lozano por financiar esta primera fase de la investigación. Así como a todos los estudiantes que participaron en las diferentes etapas del proceso de recolección de información. Asimismo, a los gerentes y responsables de seguridad y salud en el trabajo de las empresas que colaboraron en el proyecto por su disposición y tiempo para atender las entrevistas.

\section{Fuente de financiamiento}

El presente artículo es resultado del proyecto de investigación "Comparativo de la accidentalidad laboral en empresas del sector petroquímico que se encuentran certificadas en el sistema de gestión de seguridad y salud ocupacional bajo criterios OHSAS 18001:2007", financiado por la Universidad Jorge Tadeo Lozano.

\section{REFERENCIAS}

1. Zutshi A, Sohal AS. Integrated management system. The experience of three Australian organizations. J Manuf Technol Manag. 2005;16(2):211-232

2. Colombia. Gobierno Nacional. Ley No.1562 del 11 jul 2012 por la cual se modifica el sistema de riesgos laborales y se dictan otras disposiciones en materia de salud ocupacional [en línea]. [consultado mar 2016]. Disponible en: http:// wsp.presidencia.gov.co/Normativa/Leyes/Documents/ley156211072012.pdf

3. Agencia Europea de Seguridad y Salud en el Trabajo. Uso de los sistemas de gestión de la seguridad y salud en el trabajo en los Estados miembros de la Unión Europea. Facts. 2002(26):00

4. Organización Internacional del Trabajo. Sistemas de gestión de la SST: una herramienta para la mejora continua. Turín: OIT; 2011. 28 p.

5. Riaño-Casallas Ml, Molano J. Gestión de la seguridad y salud en el trabajo en hospitales públicos bogotanos de alta complejidad: una perspectiva estratégica. En: Experiencias de investigación en salud y seguridad en el trabajo. Bogotá: Universidad Nacional; 2012. p. 145.

6. Rubio Romero JC. Sistemas de gestión de la seguridad y salud en el trabajo: ¿certificables o no certificables? Directrices de la OIT vs Norma OHSAS 18001. Rev INSHT [on line] 2001 [consultado nov 2012];(14): 4-13. Disponible en: http://www.insht.es/InshtWeb/Contenidos/Documentacion/TextosOnline/Rev_ INSHT/2001/14/seccionJurTextCompl.pdf

7. Instituto Colombiano de Normas Técnicas y Certificación-ICONTEC. NTC OHSAS 18001:2007. Sistema de gestión de seguridad y salud ocupacional. Bogotá: ICONTEC; 2007. 15 p.
8. Hernández T, Godinez CLI. Procedimiento para el diseño e implantación de un sistema de gestión integrado en el Biocen. Ing Ind (La Habana). 2007;28(2):27-33.

9. Fernández-Muñiz $B$, Montes-Peón JM, Vásquez-Ordás C. Occupational risk management under the OHSAS 18001 estándar: analysis of perceptions and attitudes of certified firms. J Clean Prod. 2012;24:36-47.

10. Vinodkumar MN, Bhasi M. A study on the impact of management system certification on safety management. Safety Sci. 2011;49(3):498-507.

11. Omran A, Bakar A, Sen TH. The implementation of OHSAS 18001 in construction industry in Malasya. J Eng (Deva-Rumania). 2008;4(3):157-162.

12. Fernández-Muñiz B, Montes-Peón JM, Vásquez-Ordás C. Safety climate in OHSAS 18001-certificated organisations: Antecedents and consequences of safety behaviour. Accid Anal Prev. 2012;45:745-758.

13. Chang Jl, Liang Chiu-Lan. Performance evaluation of process safety management systems of paint manufacturing facilities. J Loss Prevent Proc . 2009;22(4):398-402.

14. Abad J, Lafuente E, Vilajosana J. An assessment of the OHSAS 18001 certification process: Objetive drivers and consequences on safety performance and labour productivity. Safety Sci. 2013;60:47-56.

15. Hamidi N, Omidvari M, Meftahi M. The effect of integrated management system on safety and productivity indices: Case study; iranian cement industries. Safety Sci. 2012;50:1180-1190. 